\title{
Improved 4D-STEM Strain Mapping Precision Using Patterned Probes
}

Steven E. Zeltmann ${ }^{1}$, Alexander Müller², Karen C. Bustillo², Benjamin H. Savitzky ${ }^{2}$, Andrew M Minor $^{1,2}$, and Colin Ophus ${ }^{2 *}$

1. Materials Science and Engineering Department, University of California Berkeley, Berkeley, CA, USA.

2. National Center for Electron Microscopy, Molecular Foundry, Lawrence Berkeley National Laboratory, Berkeley, CA, USA.

* Corresponding author: cophus@gmail.com

Mapping strain at the nanoscale with 4D-STEM relies on computational analysis of large series of diffraction patterns to precisely locate the diffracted beams and compute the local distortion of the lattice [1]. In thick samples, dynamical scattering causes intensity variations inside converged beam electron diffraction (CBED) disks that hinder precise determination of their location. This is because the usual method of cross correlation between a reference probe and the experimental CBED disks relies on similarity between the reference and the measured disks, which is lost due to such dynamical effects. Careful filtering of the data and fine tuning of the cross correlation have previously been used to make the fitting more robust to such structure, but substantial position errors can still remain [2]. Here we demonstrate the use of probes with patterning in momentum space to superimpose a regular structure onto the CBED disks and increase the amount of sharply defined edges to improve the cross correlation precision in the presence of unevenly illuminated disks, similar to the annular mask suggested by [3].

Bullseye aperture plates were fabricated by focused ion beam (FIB) milling of gold coated silicon nitride TEM windows. Approximately one gram of gold was thermally evaporated onto a $200 \mathrm{~nm}$ thick silicon nitride membrane window and a FEI Helios G4 UX was used to mill the bullseye patterns into the window. A scanning electron micrograph of the milled pattern is shown in Figure 1a. For our initial experiments, we produced bullseye apertures of 70,40,20, and $10 \mu \mathrm{m}$ diameter (chosen to match the sizes of the standard circular apertures installed in our TitanX) with 4, 3, and 2 rings pairs. Also visible on the window are a set of circular holes, which can be used to achieve nanobeam probes with smaller convergence angles than possible with the standard set.

4D-STEM maps were acquired using a FEI TitanX operated at $300 \mathrm{kV}$ on a polished $\mathrm{Si}<110>$ wedge, with the bullseye aperture plate inserted in the second condenser aperture holder. Maps were acquired from a $25 \times 25$ spot grid with $2 \mathrm{~nm}$ step spacing, acquiring a 512 × 512 pixel diffraction pattern at each scan point. The $40 \mu \mathrm{m}$ bullseye aperture with four ring pairs was used to achieve a semi-convergence angle of $\sim 3 \mathrm{mrad}$, yielding a typical CBED pattern shown in Figure 1b. For comparison we also acquired a 4D-STEM dataset from the same sample region using a standard circular aperture with a typical pattern shown in Figure 1c, and reference scans over vacuum to obtain the best quality experimental cross correlation templates.

The 4D-STEM datasets were processed using the open source py4DSTEM package which is under development at NCEM, and freely available at [4]. Hybrid correlation of the template with the experimental stack was used to locate the disk positions to single-pixel accuracy, and a set of basis vectors was estimated and used to assign indices to each disk. The disk positions were then used to obtain refined lattice vectors by least squares fitting, and the change in these lattice vectors was mapped across the scan region to calculate strain maps. 
To assess the precision of strain measurements with the bullseye probes, we performed a crossvalidation analysis by fitting a lattice to half of the disks randomly chosen from each diffraction pattern and measuring the error in the positions of the other half with respect to the fitted lattice, as shown in Figure 2a. The RMS position error from each diffraction pattern is shown in the histogram in Figure $2 b$, which shows a roughly five-fold improvement in precision. Using the bullseye probes we are able to achieve subpixel precision of the fitted lattice from a single diffraction pattern, which is not possible using the standard circular apertures.

\section{References:}

[1] Ozdal, et al., Applied Physics Letters 106(2015), 253107.

[2] Pekin, et al., Ultramicroscopy 176(2017), p. 170-176.

[3] Mahr, et al., Ultramicroscopy 158(2015), p. 38-48.

[4] https://github.com/bsavitzky/py4DSTEM

[5] S.Z. was supported by STROBE: A National Science Foundation Science \& Technology Center under Grant No. DMR 1548924. B.S was supported by Toyota Research Institute. Work at the Molecular Foundry was supported by the Office of Science, Office of Basic Energy Sciences, of the U.S. Department of Energy under Contract No. DE-AC02-05CH11231.
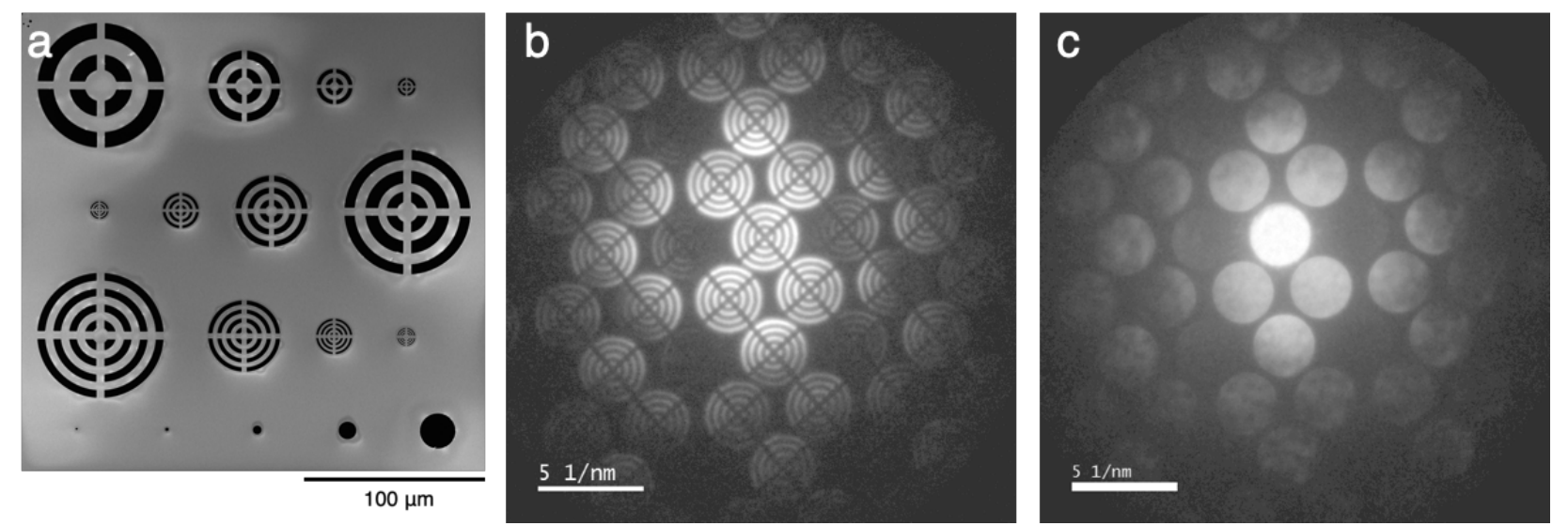

Figure 1. (a) Bullseye aperture plate with 10, 20, 40 and $70 \mu \mathrm{m}$ bullseye gratings having 2, 3, and 4 ring pairs. (b) Typical bullseye $\mathrm{Si}<110>$ CBED pattern from a 4D-STEM dataset, and (c) typical circular aperture CBED pattern from the same sample region.
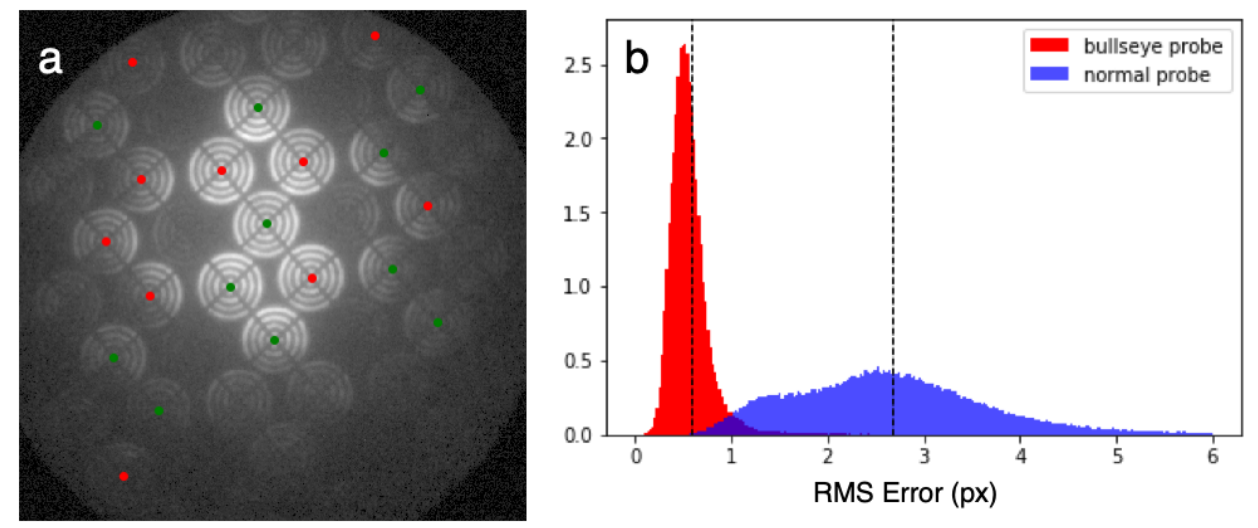

Figure 2. (a) Illustration of the cross-validation procedure. The disks marked in green are used to determine the lattice and the peaks in red are used to determine the error. (b) Cross validation results of the bullseye structured and normal probes. Vertical dashed lines show the means of the RMS errors. 